\title{
THE APPLICATION OF IRON (III) PHOSPHATE IN THE SYNTHESIS OF $N$-SUBSTITUTED PYRROLES
}

\author{
MARJAN SAMADI, FARAHNAZ K. BEHBAHANI
}

Department of Chemistry, Karaj Branch, Islamic Azad University, Karaj, Iran.

\begin{abstract}
A variety of $N$-substituted pyrroles have been prepared by reacting 2,5-hexadione with amines or diamines in the presence of iron (III) phosphate at room temperature under solvent-free conditions. The experiment protocol features simple operations, and the products are isolated in high yields (88-99\%).
\end{abstract}

Keywords: $N$-substituted pyrroles, iron (III) phosphate, 2,5-hexadione

\section{INTRODUCTION}

Substitution at nitrogen in the pyrrole ring has proved the significance of the pyrrole nucleus in various biological activities as analgesic ${ }^{1,2}$, CNS depressant $^{3}$, antifungal ${ }^{4}$, antimycobacterial ${ }^{5,6}$, anticancer ${ }^{7,8}$, anticonvulsant ${ }^{9,10}$ and anti HIV ${ }^{11}$ activities. Consequently, many methods for the synthesis of diversely substituted pyrroles have been developed such as montmorillonite, $\mathrm{KSF}^{12}$, microwave irradiation ${ }^{13,}{ }^{14}, \mathrm{Bi}\left(\mathrm{NO}_{3}\right)_{3} \cdot 5 \mathrm{H}_{2} \mathrm{O}^{15}, \mathrm{Sc}(\mathrm{OTf})_{3}{ }^{16}, \mathrm{TolSO}_{3} \mathrm{H}$ ${ }^{17}$, layered zirconium phosphate and zirconium sulfophenyl phosphonate ${ }^{18}$, titanium ${ }^{19}$ or $\mathrm{TiCl}_{4} / \mathrm{Et}_{3} \mathrm{~N}^{20}$. Some of other methods for synthesis of pyrroles include: conjugate addition reactions ${ }^{21}$, annulation reactions ${ }^{22,23}$, multicomponent reactions ${ }^{24,25}$ and aza-Wittig reactions ${ }^{26}$. However, several of these methods require prolonged reaction times, use of volatile organic solvents and toxic metals. Thus a milder, selective, non-hazardous, inexpensive, recyclable and eco-friendly catalyst is still in demand.

During the recent years, the use of reusable heterogeneous catalysts has received considerable importance in organic synthesis because of their environmental, economical and industrial aspects. The development of efficient methods using recoverable and reusable catalysts is an important goal in organic synthesis. Up to now, several reusable and heterogeneous catalysts have been designed and used. One useful example of reusable heterogeneous catalysts is iron (III) phosphate, which has been widely studied in few recent years ${ }^{27}$.

Also, one of the most common approaches to pyrroles synthesis is the PaalKnorr reaction in which 1,4-dicarbonyl compounds are converted to pyrroles in the presence of primary amines. In this communications, we wish to report an efficient, mild and green route for the Paal-Knorr synthesis of $N$-substituted pyrroles using 1,4-dicarbonyl compounds and amines in the present of $\mathrm{FePO}_{4}$ as a green and reusable catalyst (Scheme 1).

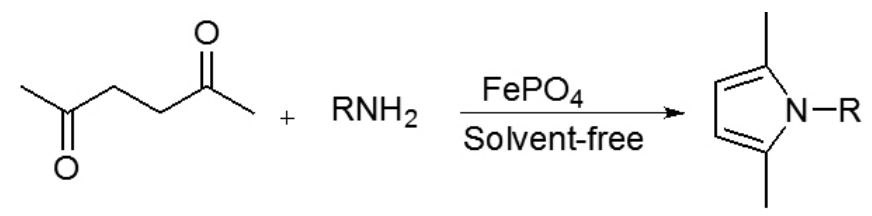

Scheme 1: $\mathrm{FePO}_{4}$-catalyzed preparation of $N$-substituted pyrroles

\section{RESULTS AND DISCUSSION}

To optimize the reaction conditions, reaction of 4-chloroaniline, 2,5-hexanedione and $\mathrm{FePO}_{4}$ was conducted in the presence of various solvents. We examined the effect of different solvents such as $\mathrm{H}_{2} \mathrm{O}, \mathrm{CH}_{2} \mathrm{Cl}_{2}$ and $\mathrm{EtOH}$ in the presence of $\mathrm{FePO}_{4}$ on a model reaction at room temperature. The results were shown in Table 1. The reaction without solvent gave the best result.
Table 1: Optimizing of the solvent in the synthesis of 1-(4-chlorophenyl)2,5-dimethyl-1H-pyrrol.

\begin{tabular}{|c|c|c|c|c|}
\hline Solvent & Free & EtOH & $\mathrm{H}_{2} \mathrm{O}$ & $\mathrm{CH}_{2} \mathrm{Cl}_{2}$ \\
\hline Yield\%* & 94 & 92 & 30 & 85 \\
\hline
\end{tabular}

* Yields of the isolated products from the reaction of 4-Chloroaniline (1 $\mathrm{mmol}), 2,5$-hexanedione $(1 \mathrm{mmol})$ and $\mathrm{FePO}_{4}(10 \mathrm{~mol} \%)$ at $10 \mathrm{~h}$.

To study this condensation reaction using the catalytic amount of $\mathrm{FePO}_{4}$, we examined the reaction yields involving of 4-chloroaniline, 2,5-hexanedione and $\mathrm{FePO}_{4}$ to afford the product under solvent-free conditions at room temperature (Table 2). As listed in Table 2, the best results were obtained at 10 $\mathrm{mol} \%$ of the catalyst under solvent-free condition and gave 1-(4-chlorophenyl)2,5-dimethyl-1H-pyrrol. The catalyst played a crucial role in the success of the reaction in terms of time and the yields. In the absence of the catalyst, the reaction could be carried out but the product was obtained in very low yield $(10 \%)$ after $48 \mathrm{~h}$.

Table 2: Optimizing of the catalyst amount in the synthesis of 1-(4chlorophenyl)-2,5-dimethyl-1H-pyrrol.

\begin{tabular}{|c|c|c|c|c|}
\hline Catalyst(mol\%) & Free & 5 & 10 & 15 \\
\hline Time(h) & 48 & 10 & 10 & 10 \\
\hline Yield\%a $^{\text {a }}$ & 10 & 55 & 94 & 94 \\
\hline
\end{tabular}

*Yields of the isolated products from the reaction of 4-Chloroaniline (1 $\mathrm{mmol}), 2,5$-hexanedione $(1 \mathrm{mmol})$ and $\mathrm{FePO}_{4}$

To generalize this optimized reaction, variety of amines were coupled with hexan-2,5-dione in the presence of a catalytic amount of $\mathrm{FePO}_{4}$ at room temperature in order to give the corresponding pyrroles in excellent yields (Table 3). The less basic aromatic amines require only slightly more time than the more basic amino compounds, and both lead to high yields of the pyrrole products. As shown in Table 3, aromatic amines with electron-donating groups or electron-withdrawing group are both effective in the Paal-Knorr reaction.

To show the merits of this catalytic method in comparison with those of reported protocols, we compiled the results of the formation of 1-phenyl))-2,5dimethyl-1H-pyrrol (Entry 1, Table 3) in the presence of a variety of catalysts. From the results given in Table 4, the advantages of our method are evident, regarding the catalyst amounts which are very important in chemical industry especially when it is combined with easy separation besides in silica sulfuric acid ${ }^{28}$.

The suggested mechanism of $\mathrm{FePO}_{4}$-catalyzed transformation is shown in Scheme 2. At the first, the carbonyl group of diketone is activated by $\mathrm{FePO}_{4}$ to obtain intermediate I. Then amine nucleophilic attacking affords intermediate II. Desired product is resulted after intramolecular nucleophilic attack of intermediate II, following dehydration of intermediate III. 
J. Chil. Chem. Soc., 60, № 2 (2015)

Table 3: Synthesis of $N$-substituted pyrroles using iron (III) phosphate as a catalyst.

\begin{tabular}{|c|c|c|c|c|c|}
\hline Entry & Amine & Product & Time (h) & Yield(\%) & M. p $\left({ }^{\circ} \mathbf{C}\right)[$ lit. $]$ \\
\hline 1 & & & 8 & 99 & $49-51[30]$ \\
\hline 2 & & & 10 & 93 & $46-48[30]$ \\
\hline 3 & & & 2 & 98 & $34-39[30]$ \\
\hline 4 & & & 6 & 88 & $43-47[30]$ \\
\hline 5 & & & 15 & 90 & $68-70[30]$ \\
\hline 6 & & & 5 & 92 & $86[30]$ \\
\hline 7 & & & 7 & 90 & $41[31]$ \\
\hline 8 & & & 3 & 97 & $110-112[30]$ \\
\hline 9 & & & 2 & 95 & $90-93[30]$ \\
\hline 10 & & & 6 & 88 & $116-119[29]$ \\
\hline 11 & & & 24 & No reaction & - \\
\hline
\end{tabular}


Table 4: Comparision of catalytic activity in the synthesis of 1-phenyl))-2,5-dimethyl-1H-pyrrol using different catalysts.

\begin{tabular}{|c|c|c|c|c|}
\hline Entry & Catalyst & $\operatorname{Tim}(\min ) /$ Temp. $\left({ }^{\circ} \mathrm{C}\right) /$ Solvent & Yield $(\%)^{*}$ & Ref \\
\hline 1 & Silica sulfuric acid (10 mol\%) & 10/r.t./Solvent-free & 95 & {$[28]$} \\
\hline 2 & KSF (excess) & 600/r.t./Solvent-free & 95 & [29] \\
\hline 3 & $\mathrm{Bi}(\mathrm{OTf})_{3}(5 \mathrm{~mol} \%)$ & $240 / 90^{\circ} \mathrm{c}$ & 85 & {$[16]$} \\
\hline 4 & $\mathrm{Bi}\left(\mathrm{NO}_{3}\right)_{3}(100 \mathrm{~mol} \%)$ & $600 /$ r.t/Solvent-free & 96 & [29] \\
\hline 5 & $\mathrm{RuCl}_{3}(5 \mathrm{~mol} \%)$ & $30 /$ r.t./Solvent-free & 94 & [29] \\
\hline 6 & $\begin{array}{l}\text { Polystyrenesulfonate( } 18 \mathrm{wt} \% \text { solution } \\
\text { in water) }\end{array}$ & $600 /$ r.t./Water-ethanol & 96 & [29] \\
\hline 7 & $\mathrm{Sc}(\mathrm{OTf})_{3}(1 . \mathrm{mol} \%)$ & 25/r.t/Solvent-Free & 93 & {$[16]$} \\
\hline 8 & $\mathrm{Y}(\mathrm{OTf})_{3}(5 \mathrm{~mol} \%)$ & 30/Room/ Solvent-free & 86 & {$[16]$} \\
\hline 9 & $\mathrm{FePO}_{4}(10 \mathrm{~mol} \%)$ & $480 /$ r.t./Solvent-free & 99 & This work \\
\hline
\end{tabular}

*Yields of the isolated products from the reaction of aniline $(1 \mathrm{mmol}), 2,5$-hexanedione $(1 \mathrm{mmol})$ and catalyst

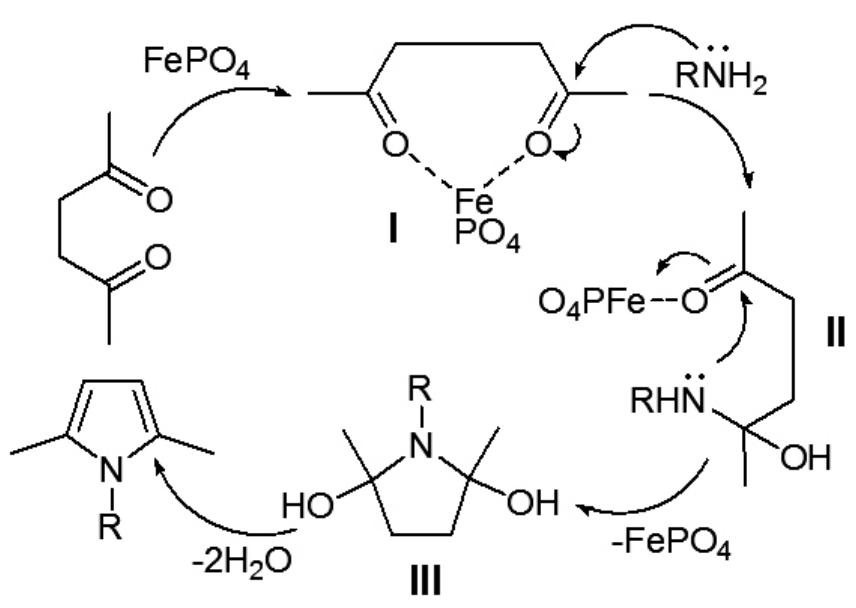

Scheme 2: The mechanism of synthesis of $N$-substituted pyrroles

\section{EXPERIMENTAL}

Melting points were measured by using the capillary tube method with an electro thermal 9200 apparatus. IR spectra were recorded on Bruker FTIR spectrometer did scanning between 4000-400 $\mathrm{cm}^{-1}$. ${ }^{1} \mathrm{HNMR}$ spectra were obtained on Bruker DRX-500MHz NMR instrument. GC/Mass spectra were recorded on an Agilent $6890 \mathrm{GC} \mathrm{Hp}-5$ capilary $30 \mathrm{~m} \times 530 \mu \mathrm{m} \times 1.5 \mu \mathrm{m}$ nominal operating at $70 \mathrm{eV}$.

Synthesis of $N$-substituted pyrroles using iron (III) phosphate. General procedure.

A solution of amine (1mmol), 2,5-hexanedione (1mmol) and $\mathrm{FePO}_{4}(10$ mol\%) was stirred under solvent-free condition and at room temperature. The progress of the reaction was followed by TLC. After completion of the reaction, ethanol $(10 \mathrm{ml})$ was added and the catalyst was removed by filtration. Evaporation of the solvent under reduced pressure gave the products.

To evaluate of reusability of the catalyst, in case of (entry 1 , Table 3 ), removed catalyst was washed with $\mathrm{CH}_{2} \mathrm{Cl}_{2}(15 \mathrm{ml})$, dried at $60{ }^{\circ} \mathrm{C}$ and was subjected for three runs. Results were yielded in $95,93,90 \%$.

Physical and spectra data

2,5-Dimethyl-1-phenyl-1H-pyrrole (Table 3, Entry 1): Cream solid, m.p. 49-5 $1^{\circ} \mathrm{C}$ [30], IR spectrum, $v, \mathrm{~cm}^{-1}: 3099(\mathrm{CH}$, Aromatic), $2927(\mathrm{CH}$, Aliphatic), $1598\left(\mathrm{C}=\mathrm{C}\right.$, Aromatic) $1519,1494,1402,1380(\mathrm{C}-\mathrm{N}) .{ }^{1} \mathrm{H}$ NMR spectrum, $\delta$, ppm $(J, \mathrm{~Hz}): 2.03\left(6 \mathrm{H}, \mathrm{s}, \mathrm{CH}_{3}\right), 5.90(2 \mathrm{H}, \mathrm{s},=\mathrm{CH}), 7.20-7.47(5 \mathrm{H}$, $\mathrm{m}, \mathrm{Ar}){ }^{13} \mathrm{C}$ NMR spectrum, $\delta$, ppm: 141.1, 129.4, 128.3, 125.6, 121.6, 109.1, 11.5. GC/Mass[ $\left.\mathrm{M}^{+}\right]$: 171.1.

1-(4-Chlorophenyl)-2,5-dimethyl-1H-pyrrole (Table 3, Entry 2): Brown solid, m.p. $46-48^{\circ} \mathrm{C}$ [30], IR spectrum, $v, \mathrm{~cm}^{-1}: 3097(\mathrm{CH}$, Aromatic), 2974(CH, Aliphatic), 1596 (C=C, Aromatic), 1496, 1369, 1321(C-N), 757(C$\mathrm{Cl}) .{ }^{1} \mathrm{H}$ NMR spectrum, $\delta$, ppm $(J, \mathrm{~Hz}): 2.02\left(6 \mathrm{H}, \mathrm{s}, \mathrm{CH}_{3}\right), 5.89(2 \mathrm{H}, \mathrm{s},=\mathrm{CH})$, 7.13-7.16 $(2 \mathrm{H}, \mathrm{d}, \mathrm{Ar}), 7.41-7.44(2 \mathrm{H}, \mathrm{d}, \mathrm{Ar}) .{ }^{13} \mathrm{C}$ NMR spectrum, $\delta, \mathrm{ppm}$ :
139.1, 131.1, 128.3, 123.6, 121.6, 109.1, 11.5. GC/Mass[M+ $\left.\mathrm{M}^{+}\right]$: 205.07.

1-(4-Methoxyphenyl)-2,5-dimethyl-1H-pyrrole ( Table 3, Entry 3): Brown solid, m.p. 34-39 ${ }^{\circ} \mathrm{C}$ [30], IR spectrum, $v, \mathrm{~cm}^{-1}: 3099$ (CH, Aromatic), 2958 (CH, Aliphatic), 1514 (C=C, Aromatic), 1461, 1440, 1367(C-N), 1043 (C-O). ${ }^{1} \mathrm{H}$ NMR spectrum, $\delta$, ppm $(J, \mathrm{~Hz}): 2.01\left(6 \mathrm{H}, \mathrm{s}, \mathrm{CH}_{3}\right), 3.85(3 \mathrm{H}, \mathrm{s}$, $\left.\mathrm{OCH}_{3}\right), 5.87(2 \mathrm{H}, \mathrm{s},=\mathrm{CH}) 6.94-6.97(2 \mathrm{H}, \mathrm{d}, \mathrm{Ar}), 7.11-7.14(2 \mathrm{H}, \mathrm{d}, \mathrm{Ar}) .{ }^{13} \mathrm{C} \mathrm{NMR}$ spectrum, $\delta$, ppm: $157.5,133.1,128.3,123.6,122.6,114.9,109.1,55.9,11.5$. $\mathrm{GC} / \operatorname{Mass}\left[\mathrm{M}^{+}\right]: 201.12$.

2,5-Dimethyl-1-p-tolyl-1H-pyrrole (Table 3, Entry 4): Brown solid, m.p. $43-47{ }^{\circ} \mathrm{C}$ [30], IR spectrum, $v, \mathrm{~cm}^{-1}: 3046(\mathrm{CH}$, Aromatic), $2983(\mathrm{CH}$, Aliphatic), $1590\left(\mathrm{C}=\mathrm{C}\right.$, Aromatic), 1515, 1436, $1380(\mathrm{C}-\mathrm{N}) .{ }^{1} \mathrm{H}$ NMR spectrum, $\delta$, ppm $(J, \mathrm{~Hz}): 2.01\left(6 \mathrm{H}, \mathrm{s}, \mathrm{CH}_{3}\right), 2.25\left(3 \mathrm{H}, \mathrm{s}, \mathrm{CH}_{3}\right), 5.87(2 \mathrm{H}, \mathrm{s},=\mathrm{CH}), 6.94-$ $6.97(2 \mathrm{H}, \mathrm{d}, \mathrm{Ar}), 7.11-7.14(2 \mathrm{H}, \mathrm{d}, \mathrm{Ar}) .{ }^{13} \mathrm{C}$ NMR spectrum, $\delta, \mathrm{ppm}: 138.5$, 135.3, 129.3, 123.6, 121.5, 109.1, 24.5, 11.5. GC/Mass[M+1: 185.12.

1-(4-Bromophenyl)-2,5-dimethyl-1H-pyrrole (Table 3, Entry 5): Brown solid, m.p. $68-70{ }^{\circ} \mathrm{C}$ [30], IR spectrum, $v, \mathrm{~cm}^{-1}: 3070(\mathrm{CH}$, Aromatic), 2981 (CH, Aliphatic), 1587 (C=C, Aromatic), 1519, 1483, 1380, 1319 (C-N), 840, 547, (C-Br). ${ }^{1} \mathrm{H}$ NMR spectrum, $\delta$, ppm $(J, \mathrm{~Hz}): 2.01\left(6 \mathrm{H}, \mathrm{s}, \mathrm{CH}_{3}\right), 5.88$ $(2 \mathrm{H}, \mathrm{s},=\mathrm{CH}), 7.05-7.58(2 \mathrm{H}, \mathrm{d}, \mathrm{Ar}), 7.55-7.85(2 \mathrm{H}, \mathrm{d}, \mathrm{Ar})$.

1-(2,4-Dimethylphenyl)-2,5-dimethyl-1H-pyrrole (Table 3, Entry 6): Brown solid, m.p. $86{ }^{\circ} \mathrm{C}$ [30], IR spectrum, $v, \mathrm{~cm}^{-1}: 3046(\mathrm{CH}$, Aromatic), 2983, 2920, 2893, 2858 (CH, Aliphatic), 1590 (C=C), Aromatic), 1515, 1436, $1380,1321(\mathrm{C}-\mathrm{N}) .{ }^{1} \mathrm{H}$ NMR spectrum, $\delta, \mathrm{ppm}(J, \mathrm{~Hz}): 2.01\left(6 \mathrm{H}, \mathrm{s}, \mathrm{CH}_{3}\right), 2.35$ $\left(6 \mathrm{H}, \mathrm{s}, \mathrm{CH}_{3}\right), 5.87(2 \mathrm{H}, \mathrm{s},=\mathrm{CH}), 6.94-6.97(2 \mathrm{H}, \mathrm{m}, \mathrm{Ar}), 7.11-7.14(1 \mathrm{H}, \mathrm{d}, \mathrm{Ar})$ ${ }^{13} \mathrm{C}$ NMR spectrum, $\delta$, ppm: $138.5,135.3,131.5,128.3,126.5,125.5,121.5$, 109.1, 26.6, 16.3, 11.5. GC/Mass[M+1 $\mathrm{M}^{+}$199.14.

1-Hexyl-2,5-dimethyl-1H-pyrrole (Table 3, Entry 7): Brown solid, m.p. $41{ }^{\circ} \mathrm{C}$ [31], IR spectrum, $v, \mathrm{~cm}^{-1}: 3060(\mathrm{CH}$, Aromatic), 2956, 2929, $2858(\mathrm{CH}$, Aliphatic), 1517 (C=C, Aromatic), 1451, 1409, 1371, 1300 (C-N), 1019(C-C). ${ }^{1} \mathrm{H}$ NMR spectrum, $\delta$, ppm $(J, \mathrm{~Hz}): 0.95\left(3 \mathrm{H}, \mathrm{t}, \mathrm{CH}_{3}\right), 1.30-1.81\left(6 \mathrm{H}, \mathrm{m}, \mathrm{CH}_{2}\right)$, $2.01\left(6 \mathrm{H}, \mathrm{s}, \mathrm{CH}_{3}\right), 3.85\left(2 \mathrm{H}, \mathrm{d}, \mathrm{NCH}_{2}\right), 5.87(2 \mathrm{H}, \mathrm{s},=\mathrm{CH}) .{ }^{13} \mathrm{C}$ NMR spectrum, $\delta$, ppm: $127.7,107.3,43.5,31.6,27.5,125.5,22.5,14.1,11.9 . \mathrm{GC} / \mathrm{Mass}\left[\mathrm{M}^{+}\right]$: 179.17.

1-(2-(2,5-Dimethyl-1H-pyrrol-1-yl)ethyl)-2,5-dimethyl-1 H-pyrrole (Table 3, Entry 8): Yellow solid, m.p. $110-112{ }^{\circ} \mathrm{C}$ [30], IR spectrum, $v$, $\mathrm{cm}^{-1}$ : 3412, 3102 (CH, Aromatic), 2970, $2730(\mathrm{CH}$, Aliphatic), $1575(\mathrm{C}=\mathrm{C}$, Aromatic), 2520, 1474, 1441, 1406, 1301 (C-N), 1015 (C-C). ${ }^{1} \mathrm{H}$ NMR spectrum, $\delta$, ppm $(J, \mathrm{~Hz}): 2.08\left(12 \mathrm{H}, \mathrm{s}, \mathrm{CH}_{3}\right), 3.95\left(4 \mathrm{H}, \mathrm{s}, \mathrm{CH}_{2}\right), 5.77(4 \mathrm{H}$, $\mathrm{s},=\mathrm{CH}) .{ }^{13} \mathrm{C}$ NMR spectrum, $\delta$, ppm: $127.7,107.0,45.5,11.9 . \mathrm{GC} / \mathrm{Mass}\left[\mathrm{M}^{+}\right]$: 216.16 .

1-(2-Hydroxyphenyl)-2, 5-dimethylpyrrole (Table 3, Entry 9): Yellow solid, m.p. $90-93{ }^{\circ} \mathrm{C}[30]$, IR spectrum, $v, \mathrm{~cm}^{-1}: 3370$ (CH, Aromatic), 2918, 2885, 2852 (CH, Aliphatic), 1589 (C=C, Aromatic), 1500, 1398, 1319, 1232 $(\mathrm{C}-\mathrm{N}), 748,621 \mathrm{~cm}^{-1} .{ }^{1} \mathrm{H}$ NMR spectrum, $\delta$, ppm $(J, \mathrm{~Hz}): 1.96(6 \mathrm{H}, \mathrm{s}), 5.95$ $(2 \mathrm{H}, \mathrm{s}), 6.97-7.00(1 \mathrm{H}, \mathrm{d}, J=7.2 \mathrm{~Hz}), 7.04-7.12(2 \mathrm{H}, \mathrm{m}), 7.29-7.34(1 \mathrm{H}, \mathrm{d})$ ${ }^{13} \mathrm{C}$ NMR spectrum, $\delta$, ppm: 149.0, 130.0, 128.3, 127.2, 123.0, 122.0, 116.5, 109.1, 11.5. GC/Mass[ $\left.\mathrm{M}^{+}\right]: 187.1$.

1-(1-Naphthalenyl)-2,5-dimethylpyrrole (Table 3, Entry 10): Brown solid, m.p. 116-119 ${ }^{\circ} \mathrm{C}$ [29], IR spectrum, $v, \mathrm{~cm}^{-1}: 3100$ (CH, Aromatic), 2972, 2926, 2880 (CH, Aliphatic), 1521, 1461, 1411, 1397, 1304 (C-N). ${ }^{1} \mathrm{H}$ NMR spectrum, $\delta, \operatorname{ppm}(J, \mathrm{~Hz}): 1.87(6 \mathrm{H}, \mathrm{s}), 5.98(2 \mathrm{H}, \mathrm{s}), 7.10-7.12(1 \mathrm{H}, \mathrm{d}, J=8.4$ $\mathrm{Hz}), 7.40-7.44(1 \mathrm{H}, \mathrm{d}, J=8.4 \mathrm{~Hz}), 7.48-7.56(2 \mathrm{H}, \mathrm{m}), 7.90-7.92(2 \mathrm{H}, \mathrm{d}, J=$ 
$8 \mathrm{~Hz}) .{ }^{13} \mathrm{C}$ NMR spectrum, $\delta$, ppm: 135.9, 134.3, 129.9, 128.3, 126.9, 126.3, 123.3, 109.1, 11.5. GC/Mass[ $\left.\mathrm{M}^{+}\right]: 221.12$.

\section{CONCLUSION}

In conclusion, $\mathrm{FePO}_{4}$ was found to be mild and effective catalyst for the Paal-Knorr reaction of 2,5-hexandione with aryl amines giving $N$-substituted pyrroles in good-to-excellent yields. The use of this inexpensive and easily available catalyst under mild conditions, the cleaner reaction and greater selectivity make this protocol practical and economically attractive.

\section{REFERENCES}

1. W. Malinka, M. S. Dziuba, G. Rajtar, A. Rubaj, Z. Kleinrok, Farmaco, 54, 390, (1999).

2. W. Malinka, M. Kaczmarz, A. Redzicka, B. Filipek, J. Sapa, Farmaco, 60, $15,(2005)$.

3. W. Malinka, M. S. Dziuba, G. Rajtar, R. Rejdak, K. Rejdak, Z. Kleinrok, Pharmazie, 55, 9, (2000).

4. D. Seref, C. K. Ahmet, K. Nuri, Eur. J. Med. Chem. 34, 275, (1999).

5. D. Delia, G. Lampis, R. Fioravanti, M. Biava, G. C. Porretta, S. Zanetti, R. Pompei, Antimicrob. Agents Chemother. 42, 3035, (1998).

6. M. Biava, F. Rossella, C. P. Giulio, D. Delia, M. Carlo, R. Pompei, Bioorg. Med. Chem. Lett. 9, 2893, (1999).

7. F. B. Miguel, F. Ascension, G. Mercedes, Chem. Pharm. Bull. 37, 2710, (1998).

8. T. C. Maria, C. Cenzo, O. Valentina. Bioorg. Med. Chem. 11, 495, (2003)

9. I. K. Sorokina, N. I. Andreeva, S. M. Golovina, Pharm. Chem. J. 23, 975, (1989).

10. J. R. Carson, R. J. Carmosin, P. M. Pitis, J. L. Vaught, H. R. Almond, J. P. Stables, H. H. Wolf, E. A. Swinyard, H. S. White, J. Med. Chem. 40, 1578, (1997).

11. J. Shibo, L. Hong, L. Shuwen, Z. Qian, H. Yuxian, K. Asim, Antimicrob. Agents Chemother 48, 4349, (2004).
12. B. K. Banik, S. Samajdar, I. Banik, J. Org. Chem. 69, 213, (2004).

13. T. N. Danks, Tetrahedron Lett. 40, 3957, (1999).

14. H. S. P. Rao, S. Jothilingam, H. W. Scheeren, Tetrahedron 60, 1625, (2004).

15. B. K. Banik, I. Banik, M. Renteria, S. K. Dasgupta, Tetrahedron Lett. 46, 2643, (2005).

16. J. Chen, H. Wu, Z. Zheng, C. Jin, X. Zhang, W. Su, Tetrahedron Lett. 47, $5383,(2006)$.

17. J. J. Klappa, A. E. Rich, K. McNeill, Org. Lett. 4, 435, (2002).

18. M. Curini, F. Montanari, O. Rosati, E. Lioy, R. Margarita, Tetrahedron Lett. 44, 3923, (2003).

19. A. Fuerstner, H. Weintritt, A. Hupperts, J. Org. Chem. 60, 66379, (1995).

20. M. Periasamy, G. Srinivas, P. Bharathi, J. Org. Chem. 64, 4204, (1999).

21. R. K. Dieter, H. Yu, Org. Lett. 2, 2283, (2000).

22. A. Arcadi, E. Rossi, Tetrahedron 54, 15253, (1998).

23. Lee, C. F.; Yang, L. M.; Hwu, T. Y.; Feng, A. S.; Tseng, J. C.; Luh, T. Y. J. Am. Chem. Soc. 122, 4992, (2000).

24. G. Dou, C. Shi, D. Shi, J. Comb. Chem. 10, 810, (2008).

25. A. R. Bharadwaj, K. A. Scheidt, Org. Lett. 6, 2465, (2004).

26. A. Katritzky, J. Jiang, P. J. Steel, J. Org. Chem. 59, 4551, (1994).

27. F. K. Behbahani, T. Yektanezhad, A. R. Khorrami, Heterocycls 81, 2313, (2010); F. K. Behbahani, M. Farahani, H. A. Oskooie, Korean J. Chem. Soc. 55, 633, (2011); F. K. Behbahani, M. Farahani, Lett. Org. Chem. 81, 436, (2011); F. K. Behbahani, P. Ziaei, Chem. Heterocycl. Compnds. 48, 1011, (2012); F. K. Behbahani, M. Homafar, Syn. React. Inorg. Met. 42, 291, (2011).

28. H. Veisi, Tetrahedron Lett. 51, 2109, (2010).

29. K. De Surya, Catal. Lett. 124, 174, (2008).

30. V. Patil, R. Sinaha, N. Masand, J. Jain, Dig. J. Nanomater. Bios. 4, 471, (2009).

31. www.chemspider.com 